\title{
On the norming constants occurring in convergent Markov chains
}

\section{Harry Cohn}

Several theorems concerning the norming constants $\left\{a_{n}\right\}$ and $\left\{b_{n}\right\}$ making a normed Markov chain $\left\{a_{n}\left(x_{n}+b_{n}\right): n \geq 0\right\}$ convergent in distribution (or in probability) are given. It is shown that if Rényi's mixing condition holds, $\lim _{n \rightarrow \infty} a_{n} / a_{n+1}=1$ and $\lim _{n \rightarrow \infty} a_{n}\left(b_{n}-b_{n+1}\right)=0$, whereas in the general case $\lim _{n \rightarrow \infty} a_{n} / a_{n+1}=\alpha$ with $\alpha \neq 0$ and $\lim _{n \rightarrow \infty} a_{n}\left(b_{n}-b_{n+1}\right)=\beta$ exist and are finite. Examples regarding maxima of independent and identically distributed random variables, random walk, and branching processes are considered.

\section{Introduction and results}

Let $(\Omega, F, P)$ be a probability space and $\left\{X_{n}: n \geq 0\right\}$ a real valued Markov chain assuming stationary transition probabilities defined on this space. Let $\mu_{0}$ be the initial probability distribution and $P(x, B)$ the transition probability function of the chain. $\mu_{0}$ is defined on the family $B$ of the borelean sets of the real line $R$ and $P(x, B)$ satisfies the conditions:

(i) $P(x, \cdot)$ is a probability on $B$ for every $x \in R$, (ii) $P(\cdot, B)$ is a Borel measurable function for each $B \in B$.

Received 15 March 1977. 
The $n$-step transition probability function is defined recursively by setting $P^{l}(x, B)=P(x, B)$ and $P^{n}(x, B)=\int P^{n-1}(x, d y) P(y, B)$. Write $\left\{\mu_{n}: n \geq 0\right\}$ for the sequence of absolute probability distributions of the chain; that is $\mu_{n}(B)=P\left(X_{n} \in B\right)$ for $B \in B$ and $n=0,1, \ldots$. Then

$$
P^{n-m}(x, B)=P\left(X_{n} \in B \mid X_{m}=x\right)
$$

almost surely with respect to $\mu_{m}$, where by an abuse of notation we have denoted by $P\left(X_{n} \in B \mid X_{m}=x\right)$ a variant of the conditional probability of the event $\left\{X_{n} \in B\right\}$ with respect to the $\sigma$-field generated by $X_{n}$.

Besides the originally defined probability measure $P$ corresponding to a Markov chain assuming the initial probability measure $\mu_{0}$ and the transition function $P(x, B)$, we shall also consider the probability measure $P_{y}$ corresponding to a chain assuming the initial probability measure $\varepsilon_{y}$ and the transition function $P(x, B)$. Here $\varepsilon_{y}$ is defined by $\varepsilon_{y}(B)=1$ if $y \in B$ and 0 if $y \notin B$ for $B \in B$.

Denote by $F_{n}^{\infty}$ the $\sigma$-field generated by the random variables $x_{n}, x_{n+1}, \ldots$ and by $T=\bigcap_{n=0}^{\infty} F_{n}^{\infty}$ the tail $\sigma$-field of the given chain. $T$ will be said to be trivial if it contains only events of probability 0 or 1. $T$ will be said to be finite if there are only a finite number of nonequivalent events in $T$, whereas $A$ and $B$ are called equivalent if $P(A \Delta B)=0, \Delta$ being the symmetric difference of two sets. Set $y_{n}=a_{n}\left(X_{n}+b_{n}\right), n=0,1, \ldots$ where $\left\{a_{n}\right\}$ with $a_{n}>0$ and $\left\{b_{n}\right\}$ are two sequences of constants.

According to Rényi [6] we shall say that a sequence of random variables $\left\{Y_{n}: n \geq 0\right\}$ is mixing with limiting distribution $F$, if $F$ is a nondegenerate distribution function such that

$$
\lim _{n \rightarrow \infty} P\left(\left\{Y_{n} \leq x\right\} \cap B\right)=F(x) P(B)
$$

for any $B \in B$ and $x$ any continuity point of $F$. 
Let $H_{n}$ be the Hahn set occurring in the Lebesgue decomposition of $\mu_{n}$ with respect to $\mu_{n-1}$, that is the set satisfying the equality

$$
\mu_{n}(B)=\mu_{n}\left(B \cap H_{n}\right)+\mu_{n}\left(B \cap H_{n}^{c}\right)
$$

for any $B \in B$, where $H_{n}^{c}$ is the complementary set of $H_{n}, \mu_{n-1}\left(H_{n}^{c}\right)=0$ and $\mu_{n}\left(\cdot \cap H_{n}\right)$ is absolutely continuous with respect to $\mu_{n-1}$.

THEOREM 1. Suppose that

(i) $\left\{Y_{n}: n \geq 0\right\}$ is mixing with limiting distribution $F$,

(ii) $\lim _{n \rightarrow \infty} \mu_{n}\left(H_{n}\right)=1$.

Then $\lim _{n \rightarrow \infty} a_{n} / a_{n+1}=1$ and $\lim _{n \rightarrow \infty} a_{n}\left(b_{n}-b_{n+1}\right)=0$.

Proof. We shall prove that $\lim _{n \rightarrow \infty} P\left(a_{n}\left(x_{n+1}+b_{n}\right) \leq x\right)=F(x)$ for $x$ any continuity point of $F$. Toward this aim, let us first notice that

$$
\begin{aligned}
P\left(a_{n}\left(x_{n+1}+b_{n}\right) \leq x\right) & =\int P\left(x_{n+1} \leq \frac{x}{a_{n}}-b_{n} \mid x_{m+1}=y\right) \mu_{m+1}(d y) \\
& =\int_{H_{m+1}}+\int_{H_{m+1}^{e}}
\end{aligned}
$$

If we write now $A_{n}=\left\{y_{n} \leq x\right\}$ and make use of (1) and (3), we obtain $\int_{H_{m+1}} P\left(X_{n+1} \leq \frac{x}{a_{n}}-b_{n} \mid X_{m+1}=y\right) \mu_{m+1}(d y)=\int_{H_{m+1}} P\left(A_{n} \mid X_{m}=y\right) \mu_{m+1}(d y)$.

Further (2) yields

(5) $\lim _{n \rightarrow \infty} \int_{H_{m+1}} P\left(X_{n+1} \leq \frac{x}{a_{n}}-b_{n} \mid X_{m+1}=y\right) \mu_{m+1}(d y)=F(x) \mu_{m+1}\left(H_{m+1}\right)$.

For the second integral in (4) we get the inequality

$$
\int_{H_{m+1}^{c}} P\left(X_{n+1} \leq \frac{x}{a_{n}}-b_{n} \mid X_{m+1}=y\right) \mu_{m+1}(d y) \leq \mu_{m+1}\left(H_{m+1}^{c}\right) \text {. }
$$


Now using (ii) in (5) and (6) and taking into account (4) we deduce that $\left\{a_{n}\left(x_{n+1}+b_{n}\right\}: n \geq 0\right.$ of converges in distribution to $F$ and the theorem follows from a well known result due to Khintchine (see Loève [4] , Corollary, p. 205).

REMARK 1 . A condition of type (ii) relating the absolute probability distributions of the chain seems necessary for the validity of Theorem 1 . Indeed, if we do not assume any restriction on $\left\{\mu_{n}\right\}$, we may have a situation when all $\mu_{m}$ and $\mu_{n}$ with $m \neq n$ are singular; that is the set of values assumed by the chain variables are pairwise almost surely disjoint. The stationarity of the transition probabilities is in such a case of no use whatsoever and we can easily imagine converging chains of this kind for which the constants $\left\{a_{n}\right\}$ and $\left\{b_{n}\right\}$ do not behave in the way described in the above theorem.

REMARK 2. Condition ( $i i$ ) may be replaced by the requirement that $\mu_{I}$ is absolutely continuous with respect to $\mu_{0}$. The proof in this case is much easier and is left to the reader. This result is related to an example givep by Rényi and Révész [7], p. 392, where they proved that if $\lim _{n \rightarrow \infty} P_{y}\left(y_{n} \leq x\right)=F(x)$ for all $x$ continuity points of $F$ and $y \in H$ with $\mu_{0}(H)=1$ and if $\lim _{n \rightarrow \infty} a_{n} / a_{n+1}=1, \lim _{n \rightarrow \infty} a_{n}\left(b_{n}-b_{n+1}\right)=0$, then $\left\{y_{n}: n \geq 0\right\}$ is mixing with limiting distribution $F$. As we have seen in Remark 1 the restriction $\mu_{0}(H)=1$ is not sufficient and was due to an oversight; in fact in their proof Rényi and Révész used that $\mu_{1}$ is absolutely continuous with respect to $\mu_{0}$ for all $n$. The latter condition can be shown to be implied by the assumption that $\mu_{1}$ is absolutely continuous with respect to $\mu_{0}$. Indeed $\mu_{0}(A)=0$ implies $\mu_{1}(A)=0$. But

$$
\mu_{I}(A)=\int P(x, A) \mu_{0}(d x)
$$

It follows that $P(x, A)=0$ almost surely, both with respect to $\mu_{0}$ and 
$\mu_{1}$. Since

$$
\mu_{2}(A)=\int P(x, A) \mu_{1}(d x),
$$

we get $\mu_{2}(A)=0$, and so on. Thus we have

\section{THEOREM 2. Suppose that}

(i) for almost all y with respect to $\mu_{0},\left\{y_{n}: n \geq 0\right\}$ converges in distribution, under the probability measure $P_{y}$, to a nondegenerate distribution function $F$,

(ii) $\mu_{1}$ is absolutely continuous with respect to $\mu_{n}$.

Then $\lim _{n \rightarrow \infty} a_{n} / a_{n+1}=1$ and $\lim _{n \rightarrow \infty} a_{n}\left(b_{n}-b_{n+1}\right)=0$ is a necessary and sufficient condition for $\left\{Y_{n}: n \geq 0\right\}$ to be mixing with limiting distribution $F$.

We shall next deal with the case when the limiting distribution function of $\left\{y_{n}: n \geq 0\right\}$ considered under the probability measure $P_{y}$ depends on $y$ when $y$ varies in the set of values assumed by $x_{0}$.

THEOREM 3. Suppose that

(i) for almost all y with respect to $\mu_{0},\left\{Y_{n}: n \geq 0\right\}$ converges in distribution, under the probability measure $P_{y}$, to a continuous and strictly increasing distribution function $F_{y}$,

(ii) $\mu_{1}$ is absolutely continuous with respect to $\mu_{0}$. Then there exist, the limits $\lim _{n \rightarrow \infty} a_{n} / a_{n+1}=\alpha$ and $\lim _{n \rightarrow \infty} a_{n}\left(b_{n}-b_{n+1}\right)=\beta$ with $-\infty<\alpha, \beta<\infty$ and $\alpha \neq 0$.

Proof. Plainly, for any $x$,

$$
P\left(y_{n} \leq x\right)=\int P\left(y_{n} \leq x \mid X_{0}=y\right) \mu_{0}(d y) \text {. }
$$

According to $(i), \lim _{n \rightarrow \infty} P\left(Y_{n} \leq x \mid X_{0}=y\right)=F_{y}(x)$ for almost all $y$ 
with respect to $\mu_{0}$ and, therefore, by the dominated convergence theorem,

$$
\lim _{n \rightarrow \infty} P\left(y_{n} \leq x\right)=F(x)=\int F_{y}(x) \mu_{0}(d y) \text {. }
$$

It is easy to check that $F$ is a continuous distribution function and hence that $\left\{Y_{n}: n \geq 0\right.$ ol converges in distribution to a nondegenerate $F$. Further by the Markov property and $(i i)$ we get

$$
\begin{aligned}
P\left(a_{n}\left(X_{n+1}+b_{n}\right) \leq x\right) . & =\int P\left(X_{n+1} \leq \frac{x}{a_{n}}-b_{n} \mid X_{1}=y\right) \mu_{1}(d y) \\
& =\int P_{y}\left(y_{n} \leq x\right) \mu_{1}(d y) \\
& =\int P_{y}\left(Y_{n} \leq x\right) \lambda_{0}(d y),
\end{aligned}
$$

where $\lambda=d \mu_{1} / d \mu_{0}$ is the Radon-Nicodym derivative of $\mu_{1}$ with respect to $\mu_{0}$. Using (i) in (9) we get that $\left\{a_{n}\left(x_{n+1}+b\right): n \geq 0\right.$ 아 converges in distribution to a nondegenerate limit distribution and the theorem now follows from Khintchine's Theorem concerning convergence of types (see Loève $[4]$, p. 203).

The assumption ( $i$ ) of Theorem 3 is not always satisfied in practice. On the other hand, to modify the initial probability distribution in order to make the chain satisfy $(i)$ is not always feasible, since some convergent properties of the chain may not be invariant under the change of the initial distribution. In such cases we need to consider conditions of a different type. The next theorem will provide a result of this kind.

THEOREM 4. Suppose that

(i) $\left\{Y_{n}: n \geq 0\right\}$ converges in probability to a random variable $Y$ assuming a nondegenerate distribution function $F$,

(ii) $\lim _{n \rightarrow \infty} \mu_{n}\left(H_{n}\right)=1$.

Then there exist the Zimits $\lim _{n \rightarrow \infty} a_{n} / a_{n+1}=\alpha$ and $\lim _{n \rightarrow \infty} a_{n}\left(b_{n}-b_{n+1}\right)=\beta$ with $-\infty<\alpha, \beta<\infty$, and $\alpha \neq 0$.

Proof. From the convergence in probability it follows that if we denote $A=\{Y \leq x\}$, then $\lim _{n \rightarrow \infty} P\left(A \Delta A_{n}\right)=0$ whenever $x$ is a continuity 
point of $F$. Therefore

$$
\lim _{n \rightarrow \infty} P\left(A_{n} \mid X_{m}=y\right)=P\left(A \mid X_{m}=y\right)
$$

almost surely with respect to $\mu_{m}$.

Further, in view of (3),

$$
P\left(A_{n} \mid X_{m}=y\right)=P\left(a_{n}\left(x_{n+1}+b_{n}\right) \leq x \mid X_{m+1}=y\right)
$$

almost surely on $H_{m+1}$.

Therefore

$$
\text { (11) } \begin{aligned}
P\left(a_{n}\left(x_{n+1}+b_{n}\right) \leq x\right)= & \int_{H_{m+1}} P\left(A_{n} \mid X_{m}=y\right) \mu_{m+1}(d y) \\
& +\int_{H_{m+1}^{c}} P\left(a_{n}\left(x_{n+1}+b_{n}\right) \leq x \mid X_{m+1}=y\right) \mu_{m+1}(d y) .
\end{aligned}
$$

On the other hand (10) yields

$$
\text { (12) } \lim _{n \rightarrow \infty} \int_{H_{m+1}} P\left(A_{n} \mid X_{m}=y\right) \mu_{m+1}(d y)=\int_{H_{m+1}} P\left(A \mid X_{m}=y\right) \mu_{m+1}(d y) \text {. }
$$

For the second integral of the right member of (1I) we get the inequality

$$
\int_{H_{m+1}^{f}} P\left(\alpha_{n}\left(x_{n+1}+b_{n}\right) \leq x \mid X_{m+1}=y\right) \mu_{m+1}(d y) \leq \mu_{m+1}\left(H_{m+1}^{c}\right) .
$$

Thus, by (11), (12), and (13) we obtain

(14) $\overline{\lim }_{n \rightarrow \infty} P\left(a_{n}\left(X_{n+1}+b_{n}\right) \leq x\right)-\lim _{n \rightarrow \infty} P\left(a_{n}\left(X_{n+1}+b_{n}\right) \leq x\right) \leq \mu_{m+1}\left(H_{m+1}^{c}\right)$.

Taking into account that the first member of this inequality does not depend on $m$ and making use of $(i i)$ we get that

$$
\lim _{n \rightarrow \infty} P\left(a_{n}\left(x_{n+1}+b_{n}\right) \leq x\right)=E_{1}(x) \text { (say) }
$$

exists for $x$ any continuity point of $F$.

Because $F_{1}$ is clearly a monotone function, to prove that it is a distribution function we need only check that $F_{1}(-\infty)=0$ and $F_{1}(\infty)=1$. 
We choose to prove that $F_{l}(\infty)=1$. To that aim let us notice that by the dominated convergence theorem

$$
\lim _{x \rightarrow \infty} \int_{H_{m+1}} P\left(A \mid X_{m}=y\right) \mu_{m+1}(d y)=\mu_{m+1}\left(H_{m+1}\right) .
$$

Taking now in (11) the limit as $n \rightarrow \infty$ and then the limit as $x \rightarrow \infty$ and using (12) and (16), we get

$$
F_{1}(\infty) \geq \mu_{m+1}\left(H_{m+1}\right),
$$

which by $(i i)$ tends to 1 as $m \rightarrow \infty$.

Next, we shall prove that $F_{1}$ is a nondegenerate distribution function. To do this it will be sufficient to show that for any interval $(a, b]$ with the property $P(Y \in(a, b])>0$ one has $F_{1}(b)-F_{1}(a)>0$, because by the assumption (i) there must exist at least two such intervals. But, as above, we can prove that

$$
F_{1}(b)-F_{1}(a) \geq \int_{H_{m+1}} P\left(y \in(a, b] \mid X_{m}=y\right) \mu_{m+1}(d y) \text {. }
$$

On the other hand, it is easy to see that

$$
\mu_{m}\left(\left\{x: P\left(y \in(a, b] \mid X_{m}=y\right)>0\right\}\right) \geq P(y \in(a, b]) \text {. }
$$

In view of the fact that $\mu_{m}$ and $\mu_{m+1}$ are subjected to the restriction (ii), (17) and (18) together yield $F_{1}(b)^{*}-F_{1}(a)>0$. Now the theorem follows from the same result of Khintchine mentioned in the proof of Theorem 3.

THEOREM 5. Suppose that

(i) $\left\{Y_{n}: n \geq 0\right\}$ with $b_{n}=0$ for all $n$ converges in probability to a random variable $Y$ with $P(Y \neq 0)>0$,

(ii) $\lim _{n \rightarrow \infty} \mu_{n}\left(H_{n}\right)=1$.

Then $\lim _{n \rightarrow \infty} a_{n} / a_{n+1}=\alpha$ with $\alpha \neq 0$ exists and is finite and if $\alpha \neq 1, y$ assumes an infinity of values. 
Proof. Notice first that the condition (i) differs from the corresponding one of Theorem 4 by the supplementary restriction $b_{n}=0$ for all $n$, whereas $Y$ is not required to have a nondegenerate distribution function. However, we can still obtain that $\lim _{n \rightarrow \infty} a_{n} / a_{n+1}=\alpha$ exists and is finite, because the whole proof of Theorem 4 can be copied here unchanged, except for Khintchine's result which can be replaced by the following

LEMMA. Suppose that $\left\{a_{n}{ }_{n}: n \geq 0\right\}$ with $\left\{a_{n}\right\}$ a sequence of constants converges in distribution to a limiting distribution function $F$ with the property $F(0)-F\left(0_{-}\right)<1$. If $\left\{a_{n}^{\prime}\right\}$ is another sequence of constants such that $\left\{a_{n}^{\prime} X: n \geq 0\right\}$ converges in distribution to a limiting distribution function $F^{\prime}$ which has the same property as that of $F$ mentioned above, then $\lim _{n \rightarrow \infty} a_{n} / a_{n}^{\prime}=\alpha$ with $\alpha \neq 0$ exists and is finite.

The proof of this lema is simpler than that of Khintchine's Theorem and is left to the reader.

Turning now to the proof of the theorem, we notice that it remains only to prove that if $\alpha \neq 1, Y$ assumes an infinity of values. If $F$ is strictly increasing and continuous in a certain interval of the real line, there is nothing to prove. Therefore, suppose that there exists a value $a$ such that $P(Y=a)>0$. According to $(i)$ we can take $a \neq 0$. By an argument similar to that used before to prove the positivity of (17) we can deduce that if $Y^{\prime}$ stands for the limit in probability of $\left\{a_{n}{ } n+1: n \geq 0\right\}$ (as $n \rightarrow \infty$ ) then $P\left(Y^{\prime}=a\right)>0$. But $Y=\alpha Y^{\prime}$, and therefore $Y$ assumes with positive probability the value aa . Further, as we have seen before, the same will hold for $Y^{\prime}$. Hence $Y$ will assume with positive probability the values $a, \alpha \alpha, \alpha^{2} \alpha, \ldots, \alpha^{n} \alpha, \ldots$, and the theorem follows.

COROLLARY. Suppose that

(i) $\left\{y_{n}: n \geq 0\right\}$ with $b_{n}=0$ for all $n$ converges in probability to a random variable $Y$ with $P(Y \neq 0)>0$, 
(ii) $\lim _{n \rightarrow \infty} \mu_{n}\left(H_{n}\right)=1$,

(iii) the tail o-field $T$ is finite.

Then $\lim _{n \rightarrow \infty} a_{n} / a_{n+1}=1$.

Proof. It suffices to notice that because $Y$ is $T$-measurable, $Y$ must assume at most a finite number of values if $T$ is finite, and then to apply Theorem 5 .

\section{Examples}

1. Any normed Markov chain converging in distribution to a nondegenerate limit distribution and assuming a trivial tail o-field for every initial distribution satisfies the assumptions of Theorem 1 . Indeed, we need $r .1 y$ check condition (ii). By a result due to Jamison and Orey (Orey [5], p. 20) for any probability measures $\lambda$ and $\nu$,

$$
\lim _{n \rightarrow \infty}\left\|\lambda P^{n}-v P^{n}\right\|=0
$$

where by $\|n\|$ we mean the norm of the signed measure $\eta$; that is $\|n\|=2 \sup _{B \in B}|n(B)|$.

If we take $\lambda=\mu_{0}$ and $\nu=\mu_{0} P$ in (19), we get $\lim _{n \rightarrow \infty}\left\|\mu_{n}-\mu_{n+1}\right\|=0$, and $(i i)$ follows.

2. The Markov chain $\left\{X_{n}: n \geq 0\right\}$ with $X_{n}=\max \left(Y_{1}, \ldots, Y_{n}\right\}$ and $\left\{Y_{n}: n \geq 0\right\}$ a sequence of independent and identically distributed random variables also satisfies the assumptions of Theorem 1 , if it converges in distribution to a nondegenerate $F$. Indeed, it is easy to see that $\left\{\mu_{n}: n \geq 0\right\}$ are equivalent measures, whereas the tail $\sigma$-field of the chain is trivial by the Hewitt-Savage $0-1$ law (see Hewitt and Savage [2]). In this case, the conclusion of Theorem 1 has been previously obtained by Richter (see Richter [8], Folgerung 7.4 and Richter [9], the proof of Satz 4). However his method does not seem extendable to a more general context, because it leans heavily on the particular form of the sequence of maxima considered. 
3. Condition (ii) of Theorem $I$ is sometimes not satisfied by some types of Markov chains for which nevertheless its conclusion holds. Take for example the random walk case, that is $X_{n}=Y_{I}+\ldots+Y_{n}$, where $\left\{Y_{n}: n \geq 1\right\}$ is a sequence of independent and identically distributed random variables. The tail $\sigma$-field of such a sequence is also trivial by the Hewitt-Savage above mentioned 0 - 1 law, but may fail to stay trivial under a different initial distribution. Indeed if $Y_{i}$ is Bernoulli distributed with values -1 and +1 assuming the probabilities $p$ and $q$ then we can easily see that $\mu_{n}$ and $\mu_{n+l}$ are singular for each $n$. However we can modify the initial distribution of the chain by taking instead a measure assuming positive probability for any state $i=\ldots,-1,0,1, \ldots$. The new Markov chain obtained in this way is also mixing with the same limiting distribution $F$, but now $\left\{\mu_{n}: n \geq 0\right\}$ are equivalent measures and condition (ii) of Theorem 1 is satisfied.

4. We shall next consider examples pertaining to Theorems 3 and 4. Let $\left\{z_{n}: n \geq\right.$ 아 be a simple branching process and $\left\{p_{i}: i \geq 0\right\}$ its offspring distribution. It has been shown that if $m=\sum_{i=1}^{\infty} i p_{i}>1$, then there exist some norming constants $\left\{C_{n}\right\}$ such that $\left\{z_{n} / C_{n}\right\}$ converges almost surely to a nondegenerate limit (see Seneta [10] and Heyde [3]), whereas $\lim _{n \rightarrow \infty} C_{n} / C_{n-1}=m$. Recently some properties asserting the convergence in distribution of $\left\{\log \left(z_{n}+1\right) / C_{n}\right\}$ for suitably chosen constants $\left\{C_{n}\right\}$ were found in the case $m=\infty$ (Darling [1] and Seneta [11]).

Because $\left\{\operatorname{Iog}\left(z_{n}+1\right): n \geq 0\right.$ 의 is also a Markov chain with stationary transition probabilities, it raises the question whether $\lim _{n \rightarrow \infty} C_{n+1} / C_{n}=\alpha$ for a certain $\alpha>0$. This question can be answered in the positive in the case when $z_{0}=I$ almost surely, and $\left\{\log \left(z_{n}+1\right) / C_{n}\right\}$ converges in distribution to a continuous and strictly increasing distribution function (Seneta [11]). Indeed, as before we can choose a new initial distribution 
$\mu_{0}$ such that $\mu_{0}(i)>0$ for all $i=0,1, \ldots$. Then $P_{i}\left(\log \left(z_{n}+1\right) / C_{n} \leq x\right)$ converges in distribution to the convolution of $i$ random variables, each of them assuming the distribution function $\lim _{n \rightarrow \infty} P\left(\log \left(z_{n}+1\right) / C_{n} \leq x\right)$ and the conditions of Theorem 3 are satisfied. We mention that results of such a kind are proved in the branching processes literature by complicated analytical methods involving the study of the iterates of the generating function.

REMARK. It is interesting to note by comparing the results and examples given above that a Markov chain satisfying Rényi's mixing condition, and in particular one assuming a trivial tail $\sigma$-field may admit in general a slower convergence rate than a Markov chain of a different type, if the chains converge in the sense mentioned in the conditions considered here. However, one should not conclude that a Markov chain cannot grow more quickly than geometrically, because a counterexample of this assertion would be provided by the branching processes with infinite mean for which $\left\{\log \left(z_{n}+1\right\} / C_{n}\right\}$ converges to a nondegenerate limiting distribution (Example 4). The rates of growth stated by the results given here pertain only to the case when convergence in distribution (or probability) holds.

\section{Referencess}

[1] D.A. Darling, "The Galton-Watson process with infinite mean", $J$. Appl. Probability 7 (1970), 455-456.

[2] Edwin Hewitt and Leonard J. Savage, "Symmetric measures on cartesian products", Trans. Amer. Math. Soc. 80 (1955), 470-501.

[3] C.C. Heyde, "Extension of a result of Seneta for the supercritical Galton Watson process", Ann. Math. Statist. 41 (1970), 739-742.

[4] Michel Loève, Probability theory, third edition (Van Nostrand, Princeton, New Jersey; Toronto; New York; London; 1963).

[5] Steven Orey, Lecture notes on limit theorems for Markov chain transition probabilities (Van Nostrand Reinhold, London, New York, Cincinnati, Toronto, Melbourne, 1971). 
[6] A. Rényi, "On mixing sequences of sets", Acta Math. Acad. Sci. Hungar. 9 (1958), 215-228.

[7] A. Rényi and P. Révész, "On mixing sequences of random variables", Acta Math. Acad. Sci. Hungar. 9 (1958), 389-393.

[8] W. Richter, "Zu einigen Konvergenzeigenschaften von Folgen zufälliger Elemente", Studia Math. 25 (1965), 231-243.

[9] Wolfgang Richter, "Das Null-Eins-Gesetz und ein Grenzwertsatz für zufällige Prozesse mit diskreter zufälliger Zeit", Wiss. Z. Techn. Univ. Dresden 14 (1965), 497-504.

[10] E. Seneta, "On recent theorems concerning the supercritical GaltonWatson process", Arn. Math. Statist. 39 (1968), 2098-2102.

[11] E. Seneta, "The simple branching process with infinite mean. I", $J$. Appl. Probability 10 (1973), 206-212.

Department of Statistics, Institute of Advanced Studies, Australian National University, Canberra, ACT. 